\title{
Mapping Migraine-Specific Quality of Life to Health State Utilities in Patients Receiving Rimegepant
}

\author{
Karissa M. Johnston (D) - Gilbert L'Italien · Evan Popoff • \\ Lauren Powell · Robert Croop · Alexandra Thiry · Linda Harris • \\ Vladimir Coric $\cdot$ Richard B. Lipton
}

Received: July 15, 2021 / Accepted: August 18, 2021 / Published online: August 29, 2021

(C) The Author(s) 2021

\section{ABSTRACT}

Introduction: Migraine is a debilitating neurological condition, affecting up to $15 \%$ of Americans. Recent estimates from a long-term safety study of rimegepant showed evidence of decreased monthly migraine days (MMD) in people with episodic migraine treated with rimegepant $75 \mathrm{mg}$. The objective of this study was to characterize migraine-specific quality of life version 2.1 (MSQv2) scores and corresponding mapped EuroQol-5 Dimensions-3 Level (EQ-5D-3L) utility values.

Methods: Study participants were randomized into two treatment regimens: individuals with 2-14 MMD received rimegepant $75 \mathrm{mg}$ as needed (PRN), and those with 4-14 MMD at baseline who received rimegepant on a fixed everyother-day schedule plus an as needed dose on

K. M. Johnston $(\varangle) \cdot$ E. Popoff · L. Powell Broadstreet Health Economics and Outcomes Research, 203-343 Railway St., Vancouver, BC V6A 1A4, Canada

e-mail: kjohnston@broadstreetheor.com

K. M. Johnston

Memorial University, St John's, NL, Canada

G. L'Italien · R. Croop · A. Thiry · L. Harris .

V. Coric

Biohaven Pharmaceuticals, New Haven, CT, USA

R. B. Lipton

Albert Einstein College of Medicine, Bronx, NY, USA days they did not treat (QOD + PRN). MSQv2 was mapped to EQ-5D-3L utilities using a validated algorithm. Outcomes were assessed for the PRN arm at baseline weeks 12, 24, 36, and 52 and for the QOD + PRN arm at baseline and week 12.

Results: At baseline, MSQv2 data were available for 1,800 patients: 1,033 with 2-8 MMD in the PRN group, 481 with 9-14 MMD in the PRN group, and 286 with 4-14 MMD in the QOD + PRN group. For all MSQv2 domains as well as mapped utility values, outcomes improved over each study visit. At baseline, EQ5D-3L utilities were $0.66,0.63$, and 0.65 for the 2-8 MMD PRN, 9-14 MMD PRN, and 4-14 MMD QOD + PRN groups, respectively. At endof-study, utilities had increased by $+0.09,+0.10$, and +0.12 for the three groups, respectively $(p<0.001$ for all comparisons with baseline). Similar trends in improvement were observed across MSQv2 subdomains; all differences were statistically significant.

Conclusions: Rimegepant $75 \mathrm{mg}$, which has been shown to be associated with reduced MMD, is associated with improvement in MSQv2 domains over time, leading to estimated improvement in EQ-5D-3L utilities. While this improvement was observed in all patientgroups, it was most pronounced in those with higher MMD and those taking rimegepant QOD + PRN. 
Trial registration: Clinical NCT03266588.

Keywords: Migraine; Patient-reported outcome; Migraine-specific quality of life (MSQv2); Preference-based instrument; EQ-5D; Utility; Mapping

\section{Key Summary Points}

In this post-hoc analysis of a long-term safety study of rimegepant $75 \mathrm{mg}$ in patients with migraine, the migrainespecific quality of life version 2.1 (MSQv2) survey was collected and mapped to EuroQol five-dimension (EQ-5D) utilities via a validated algorithm.

Patients were stratified into enrollment groups consisting of 52-week as-needed (PRN) rimegepant treatment for patients with 2-8 and 9-14 monthly migraine days (MMD), respectively, and 12-week PRN + every second day (QOD) treatment for patients with 4-14 MMD.

Over the course of follow-up, MSQv2 and mapped utilities improved across all enrollment groups.

Across enrollment groups, frequency of MMD decreased over the rimegepant treatment period, and descriptive associations were noted between lower MMD and better health-related quality of life (HRQoL), as characterized by MSQv2 and EQ-5D utility scores.

The analysis described here supports the relationship between reduction in migraine events and improved quality of life, and the impact of rimegepant (both PRN-only and QOD + PRN) on reducing frequency of MMD and increasing quality of life over time.

\section{Trials INTRODUCTION}

Migraine is a common primary headache disorder associated with significant disability $[1,2]$. This neurological condition is characterized by recurrent attacks of head pain that is typically unilateral and throbbing, and associated with a range of symptoms that include nausea, vomiting, phonophobia, and photophobia [1-3]. Health-related quality of life (HRQoL) is negatively affected in patients with migraine, and this impact increases with migraine severity and frequency [4-8]. Lower HRQoL among individuals with migraine reflects the quality of live burden on headache days as well as the burden between attacks (inter-ictal burden) which arises from disrupted planning and anticipatory anxiety among other factors $[9,10]$.

Currently, there is no single gold-standard method to measure the patient experience of migraine and its impact on HRQoL. Generic preference-based measures such as the EuroQol five-dimension (EQ-5D) or Health Utility Index (HUI) have been used to quantify HRQoL, but may not fully characterize the clinical features or burden of migraine [11]. Disease-specific measures such as the Migraine-Specific Quality of Life version 2.1 (MSQv2) and Headache Impact Test (HIT-6) are thought to have better ability to detect change associated with treatment, and are recommended for use in clinical trials for migraine therapies, particularly those with preventive effects $[12,13]$.

While disease-specific measures may be a more valid indicator of treatment success, these cannot be used directly to calculate quality-adjusted life years (QALYs) in cost-utility models, which are increasingly being used to inform policy decisions and drug reimbursements [14]. To facilitate the economic evaluation of novel migraine therapies, generic preference-based HRQoL measures are required to compare utility values over time, across treatments [14]. In migraine trials that did not directly measure health-state utilities, mapping algorithms can be used to estimate EuroQol 5-Dimensions 3-Level (EQ-5D-3L) scores from MSQv2 diseasespecific HRQoL scores, using mapping by 
statistical association, as described by Gillard et al. [14].

Rimegepant, a small molecule calcitonin gene-related peptide (CGRP) receptor antagonist, has demonstrated efficacy (at a dose of $75 \mathrm{mg}$ ) as both an acute and preventive treatment for migraine, in adults with a range of baseline migraine frequency and severity $[15,16]$. In a long-term, open-label safety study of rimegepant taken either to treat acute attacks as needed (PRN) or every other day plus PRN (QOD + PRN; Study 201; NCT03266588), the impact of rimegepant $75 \mathrm{mg}$ on HRQoL over time was measured using the MSQv2 [17]. By week 12, rimegepant-treated patients showed clinically and statistically significant improvements in mean MSQv2 scores with a mean change from baseline of +14.5 for role restrictive (RR), +11.5 for role preventive (RP), and +15.4 for emotional function (EF) $(p<0.0001)$ [17]. The observed HRQoL improvement with rimegepant $75 \mathrm{mg}$ was rapid and durable over the 52-week study period [17]. In placebo-controlled trials of rimegepant as an acute therapy, a significant reduction was observed in need for rescue medication $(18.2 \%$ vs. $32.4 \%$ of placebo patients).

To facilitate future economic analyses of rimegepant $75 \mathrm{mg}$, the objectives of the current study were to (1) map long-term MSQv2 outcomes from Study 201 to EQ-5D-3L health state utilities using the validated mapping algorithm by Gillard et al. [14], and (2) compare health state utilities across rimegepant $75 \mathrm{mg}$ dosing regimens (PRN or QOD + PRN) and by observed reduction in MMD.

\section{METHODS}

\section{Summary of Study 201}

The objective of Study 201 was to evaluate the long-term safety of rimegepant $75 \mathrm{mg}$ and to assess the effects of repeated dosing of rimegepant on MSQv2, and MMD, amongst other endpoints [17-19]. Three groups of study participants were randomized into two treatment regimens: individuals with 2-8 (Group 1) and 9-14 (Group 2) moderate to severe MMD [as defined by the International Headache Society (HIS) criteria] [20], respectively, self-administered rimegepant $75 \mathrm{mg}$ up to once daily as needed (PRN) for 52 weeks; while individuals with 4-14 (Group 3) moderate to severe MMD self-administered rimegepant $75 \mathrm{mg}$ on a fixed every-other-day schedule and as needed on non-scheduled dosing days (QOD + PRN) for 12 weeks [18].

\section{Patients and Data Collection}

Male and female episodic migraine patients, $\geq 18$ years of age, who had at least a 1 -year history of migraine (with or without aura) diagnosed as per IHS criteria were eligible [18]. Patients were required to have an age of onset of migraine prior to age 50, between 2 and 14 episodes per month lasting $4-72 \mathrm{~h}$ if untreated, and ability to distinguish migraine attacks from tension or cluster headaches. Exclusion criteria were as follows: history of basilar migraine or hemiplegic migraine, history of HIV disease, history of uncontrolled, unstable, or recently diagnosed cardiovascular disease, uncontrolled hypertension or diabetes, history of gastric or small intestinal surgery, body mass index $(\mathrm{BMI}) \geq 30$, or $\mathrm{HbA} 1 \mathrm{c} \geq 6.5$.

Patients in the QOD + PRN group were followed for 12 weeks while those in the PRN groups were followed for 52 weeks. Migraine events, tablet utilization, and adherence were recorded in patient diaries.

MSQv2 was assessed at baseline and week 12 for the QOD + PRN group, and at baseline and at weeks 12, 24, 36, and 52 for the two PRN groups.

Study 201 was conducted in accordance with Good Clinical Practice guidelines as defined by the International Conference on Harmonisation and in accordance with all applicable local regulations. The protocol was approved by centralized and local Institutional Review Boards, and participants provided written informed consent before they were screened for the study. 


\section{Description of MSQv2}

The MSQv2 is a validated 14-question diseasespecific patient-reported outcome (PRO) measure that is frequently used to measure the impact of migraine and multidimensional aspects of preventive treatments' effectiveness on HRQoL in a meaningful way $[1,21]$. The MSQv2 has three HRQoL dimensions: RR, RP, and EF [22]. The RR dimension has seven items and measures migraine impact on normal activities such as work, productivity, daily activities, energy, tiredness, concentration, leisure, and relationships [14, 23]. The RP dimension has four items and measures the impact of migraine on interrupting performance of normal activities [14]. The EF dimension has three items and measures migraine's effect on emotional function [14]. The questionnaire consists of 14 questions about migraine attacks during the past 4 weeks, with six response options at each question, ranging from 1 to $6(1$, none of the time; 2 , a little bit of the time; 3 , some of the time; 4 , a good bit of the time; 5 , most of the time; and 6, all of the time), and scored linearly on a 0-100 scale where higher scores indicate better HRQoL $[23,24]$. The minimum clinically important difference for within-group analyses are 5 points, $5-7.9$ points, and $8-10.6$ points for $\mathrm{RR}, \mathrm{RP}$, and EF, respectively [25].

\section{Description of EQ-5D-3L}

The EQ-5D-3L is a widely used generic measure of health status used for economic assessments [26]. Health states are measured for the day the questionnaire is administered, and the EQ-5D version that Gillard et al., used consists of five dimensions (1, Mobility; 2, Self-care; 3, Usual activities; 4, Pain/Discomfort; and 5, Anxiety/ Depression) and three levels (1, no problems; 2 , some problems; and 3 , extreme problems) $[14,26]$. Scores are converted to a utility value or index score by using country-specific value sets to apply preference weights $[14,26]$. The United Kingdom (UK) value set was applied by Gillard et al. to a dataset sourced from the International Burden of Migraine Study, which included participants from five Western
European countries, as well as from Australia, Brazil, Canada, Taiwan, and the United States (US) [14]. Scores range from less than 0 to 1 , where negative values are health states worse than death, 0 is equivalent to death, and 1 is perfect health [26].

\section{Utility Mapping}

Using patient-level data from Study 201, MSQv2 values were mapped to EQ-5D-3L utilities using a validated mapping by statistical association algorithm using the method described by Gillard et al. [14]. The model defined for episodic migraine was used and followed the form:

$$
\begin{aligned}
\mathrm{EQ} \_5 \mathrm{D}= & 0.2858+0.0029^{*} \mathrm{MSQ} \_\mathrm{RP} \\
& +0.0001^{*} \mathrm{MSQ} \text { _RR } \\
& +0.0027^{*} \mathrm{MSQ} \_\mathrm{EF}
\end{aligned}
$$

This validated algorithm produced statistically significant correlation coefficients between EQ-5D-3L utility scores and MSQv2 scores [14]. MSQv2 has been validated for use in patients with migraine [21], and several MSQv2 and EQ-5D-3L dimensions overlap with regards to content [14]. There were negligible missing MSQv2 data from Study 201, therefore no adjustments were made in this regard. Values were compared between baseline and end-ofstudy using paired $t$ tests, in addition to visual comparison. All analyses were stratified by study enrollment group.

\section{RESULTS}

At baseline, MSQv2 data were available for 1800 patients: 1033 with 2-8 MMD in the PRN group, 481 with 9-14 MMD in the PRN group, and 286 with 4-14 MMD in the QOD + PRN group [18]. The median age of participants was 43 years and $3.6 \%$ were 65 years of age or older, with interquartile range $33-51$ years and a maximum age of 83 years [18]. More than $80 \%$ of participants were white and $90 \%$ were female (Table 1).

MMD (SD) at baseline was 7.02 (3.74 for the PRN 2-8 group, 12.2 (4.64) for the PRN 9-14 group, and 9.0 (3.90) for the QOD + PRN 4-14 
Table 1 Baseline characteristics in Study 201

\begin{tabular}{llll}
\hline Characteristic & \multicolumn{2}{l}{ Enrollment groups } & \\
\cline { 2 - 4 } & $\mathbf{2 - 8}$ MMD PRN & 9-14 MMD PRN & 4-14 MMD QOD + PRN \\
\hline$n$ & 1033 & 481 & 286 \\
Age (years) mean (SD) & $43.47(11.79)$ & $41.89(12.39)$ & $40.56(12.7)$ \\
Female $n$ (\%) & $917(88.77)$ & $444(92.31)$ & $248(86.71)$ \\
White $n$ (\%) & $847(81.99)$ & $394(81.91)$ & $234(81.82)$ \\
Hispanic $n$ (\%) & $99(9.58)$ & $54(11.23)$ & $24(8.39)$ \\
Height (cm) mean (SD) & $165.77(8.09)$ & $165.51(8.18)$ & $166.44(8.22)$ \\
Weight (kg) mean (SD) & $83.59(22)$ & $81.71(22.89)$ & $69.81(11.66)$ \\
BMI mean (SD) & $30.42(7.76)$ & $29.74(7.73)$ & $25.16(3.46)$ \\
MMD (absolute) mean (SD) & $7.02(3.74)$ & $12.23(4.64)$ & $9.03(3.9)$ \\
MMD (normalized) mean (SD) & $7.00(3.75)$ & $12.62(4.71)$ & $9.1(4.03)$ \\
\hline
\end{tabular}

BMI body mass index; $\mathrm{cm}$ centimeter; kg kilogram; MMD monthly migraine days; $n$,number of participants per group; SD standard deviation

group. Following treatment with rimegepant (52 weeks for the PRN groups and 12 weeks for the QOD + PRN group), MMD were reduced by
0.47 (4.89) for the PRN 2-8 group, by 2.94 (5.95) in the PRN 9-14 group, and by 3.31 (3.51) in the QOD + PRN 4-14 group (Table 2). The mapping

Table 2 Monthly migraine days, Migraine-Specific Quality of Life Questionnaire v2, and EuroQol five-dimension threelevel utility at baseline and change from baseline ( 52 weeks for the PRN groups and 12 weeks for the QOD + PRN group)

\begin{tabular}{llll}
\hline & $\begin{array}{l}\text { 2-8 MMD PRN } \\
(\boldsymbol{n}=\mathbf{1 0 3 3})\end{array}$ & $\begin{array}{l}\text { 9-14 MMD PRN } \\
(\boldsymbol{n}=\mathbf{2 8 6})\end{array}$ & $\begin{array}{l}\text { 4-14 MMD QOD + PRN } \\
(\boldsymbol{n}=\mathbf{4 8 1})\end{array}$ \\
\hline MMD at baseline & $7.02(3.74)$ & $12.23(4.64)$ & $9.03(3.90)$ \\
MMD change from baseline & $-0.47(-0.84$, & $-2.94(-3.65$, & $-3.31(-3.75,-2.87)$ \\
& $-0.11)$ & $-2.24)$ & \\
MSQv2- RP at baseline & $69.28(20.45)$ & $64.47(20.75)$ & $68.06(19.73)$ \\
MSQv2-RP change from baseline & $13.78(12.30,15.26)$ & $15.60(13.17,18.04)$ & $18.98(16.44,21.51)$ \\
MSQv2-RR at baseline & $54.56(18.25)$ & $48.70(18.44)$ & $52.26(17.38)$ \\
MSQv2-RR change from baseline & $16.82(15.31,18.33)$ & $19.86(17.49,22.23)$ & $24.27(21.94,26.61)$ \\
MSQv2-EF at baseline & $63.70(25.62)$ & $54.87(26.23)$ & $60.84(25.47)$ \\
MSQv2-EF change from baseline & $16.72(14.83,18.60)$ & $19.49(16.21,22.77)$ & $24.02(20.95,27.08)$ \\
Mapped EQ-5D-3L utility at baseline & $0.66(0.12)$ & $0.63(0.12)$ & $0.65(0.11)$ \\
$\begin{array}{c}\text { Mapped EQ-5D-3L utility change } \\
\text { from baseline }\end{array}$ & $0.09(0.08,0.10)$ & $0.10(0.09,0.11)$ & $0.12(0.11,0.14)$ \\
\hline
\end{tabular}

Baseline values are mean (SD), CFB values are mean (95\% CI)

EF emotional function; MMD monthly migraine days; MSQv2 migraine-specific quality of life; $n$ number of participants in each group; RP role preventive; RR role restrictive; $\mathrm{SD}$ standard deviation 

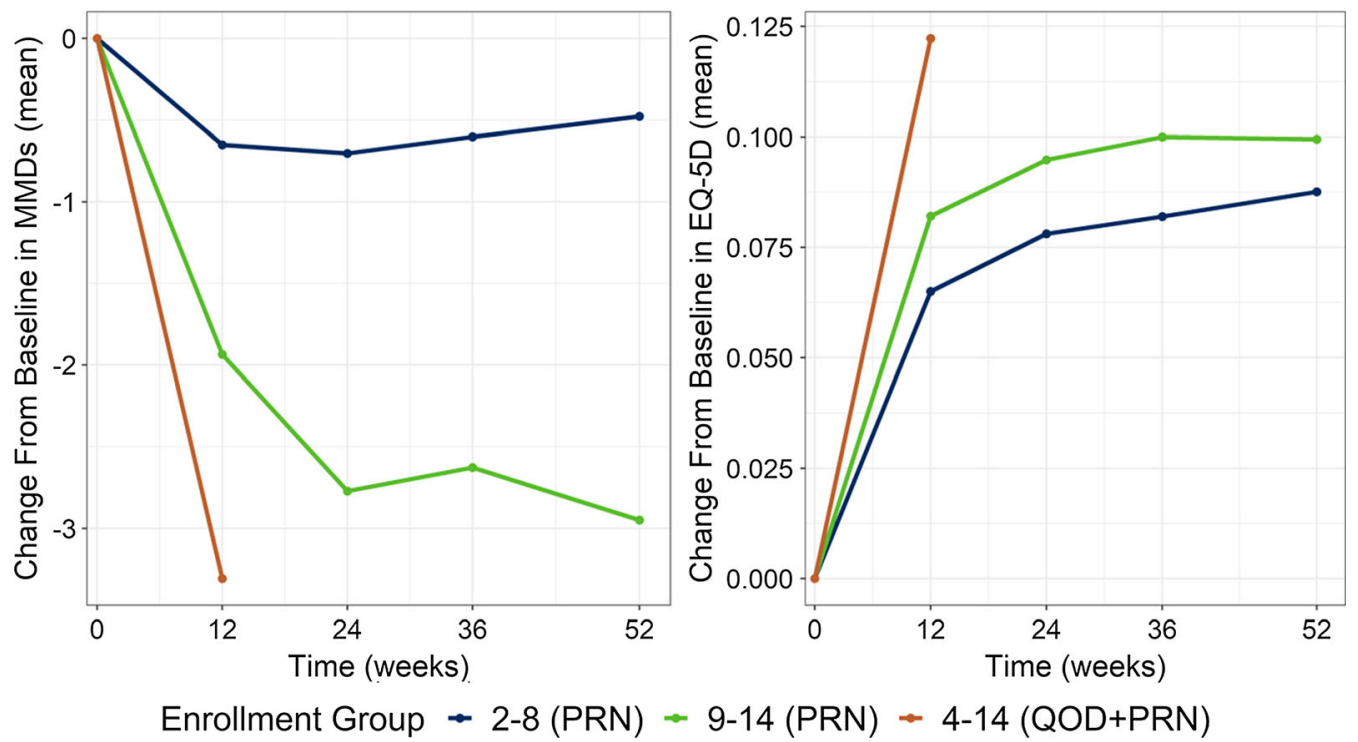

Fig. 1 Absolute monthly migraine days over time in Study 201, stratified by treatment group. The QOD + PRN enrollment group only contributed data to Week 12, and

thus Week 24, 36, and 52 data are only available for PRN enrollment groups. MMD monthly migraine days; PRN as needed; QOD every other day

study found that the groups with the greatest decrease in MMD had the highest increase in EQ-5D-3L (Fig. 1; Table 2).

All MSQv2 domains improved over time (Table 2; Fig. 2). Similar trends in improvement

were observed across MSQv2 subdomains, and all differences were statistically significant. When the MSQv2 was mapped to EQ-5D-3L utilities, baseline utility values were $0.66,0.63$, and 0.65 for the 2-8 MMD PRN, 9-14 MMD

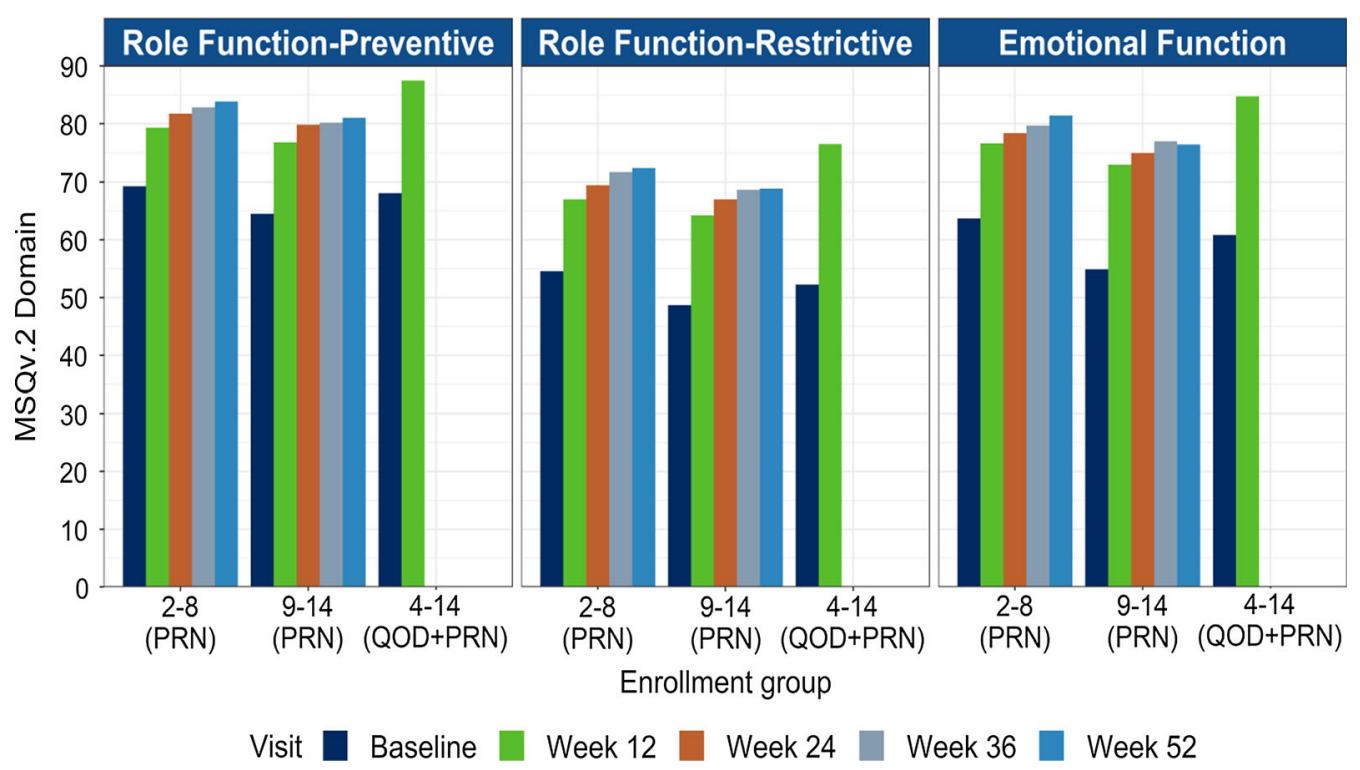

Fig. 2 MSQv2 domains over time in Study 201, stratified by treatment group; $p<0.001$ for all outcomes in all enrollment groups over time. The QOD + PRN

enrollment group only contributed data to Week 12 , and thus Week 24, 36, and 52 data are only available for PRN enrollment groups 


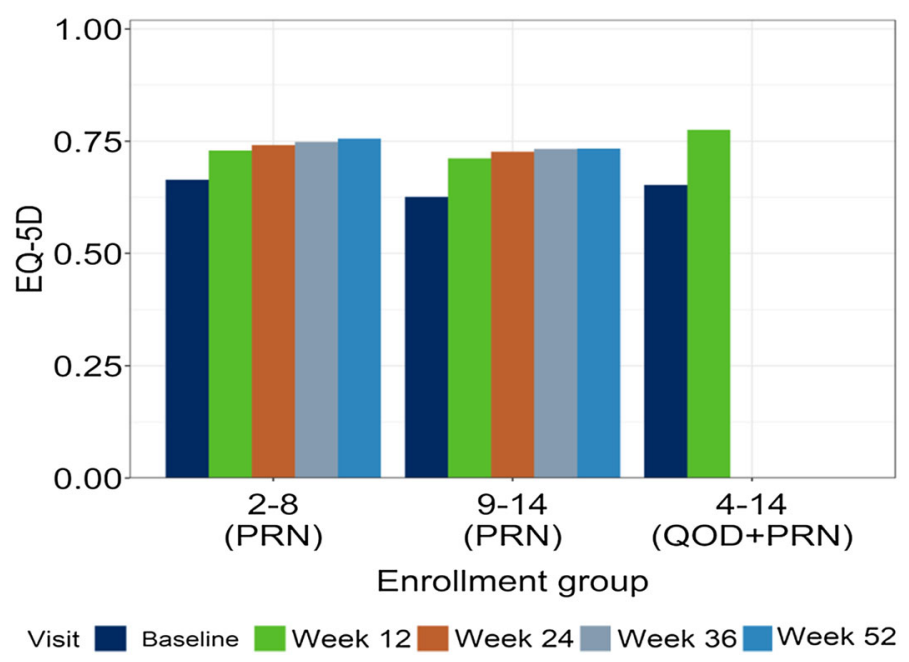

Fig. 3 EQ-5D-3L utilities mapped from MSQv2 data; $p<0.001$ for all outcomes in all enrollment groups over time. The QOD + PRN enrollment group only contributed data to Week 12, and thus Week 24, 36, and 52

PRN, and 4-14 MMD QOD + PRN groups, respectively. At end-of-study, utilities had increased by $+0.09,+0.10$, and +0.12 for the three groups, respectively $(p<0.001$ for all comparisons with baseline) (Fig. 3). In addition to the cohort-level trend of increased EQ-5D and lower MMD frequency over time, the relationship between EQ-5D and MMD is observed at the individual patient level (Fig. 4). Within all three enrollment groups, trends were observed consistently across individuals in all three enrollment groups: an inverse correlation was observed between MMD and mapped EQ$5 \mathrm{D}$ values at baseline and a greater reduction in MMD over the study period was associated with consistently associated with greater increase in EQ-5D-3L over the study period (Fig. 4).

\section{DISCUSSION}

This study provides new information about the effects of different rimegepant dosing regimens on MMD, MSQv2, and EQ-5D-3L utility scores which may be useful for economic evaluations and decision-making. Results from Study 201 showed that rimegepant $75 \mathrm{mg}$ reduced MMD and increased MSQv2 scores in three enrollment groups: 2-8 MMD PRN, 9-14 MMD PRN, data are only available for PRN enrollment groups. MSQv2 migraine-specific quality of life; PRN as needed; QOD every other day

and 4-14 MMD QOD + PRN groups, respectively. When MSQv2 scores were mapped to EQ$5 \mathrm{D}-3 \mathrm{~L}$, there was an associated improvement in EQ-5D-3L utilities. In the current study, EQ-5D$3 \mathrm{~L}$ utility scores at baseline were $0.66,0.63$, and 0.65 for the 2-8 MMD PRN, 9-14 MMD PRN, and 4-14 MMD QOD + PRN groups, respectively. At the end of Study 201, as MMD decreased with rimegepant treatment, MSQv2 increased and EQ-5D-3L utilities increased to $0.75,0.73$, and 0.77 for the three enrollment groups, resulting in change scores from baseline of $+0.09,+0.10$, and +0.12 , respectively.

Similar mappings have been conducted for the preventive migraine treatment erenumab. Di Tanna et al., also used the algorithms developed by Gillard et al., and several models to map MSQv2 to EQ-5D-3L, using data from three erenumab trials, and found a non-linear association between increased MMD and decreased EQ-5D-3L [27]. Utility values were consistently higher in patients treated with erenumab compared to those treated with placebo after adjusting for MMD, suggesting a treatment effect in addition to what is captured with MMD [27]. This limitation of MMD to not fully capture the residual impact of migraine treatment on non-migraine days was mentioned by Lipton et al. [28] in an economic 

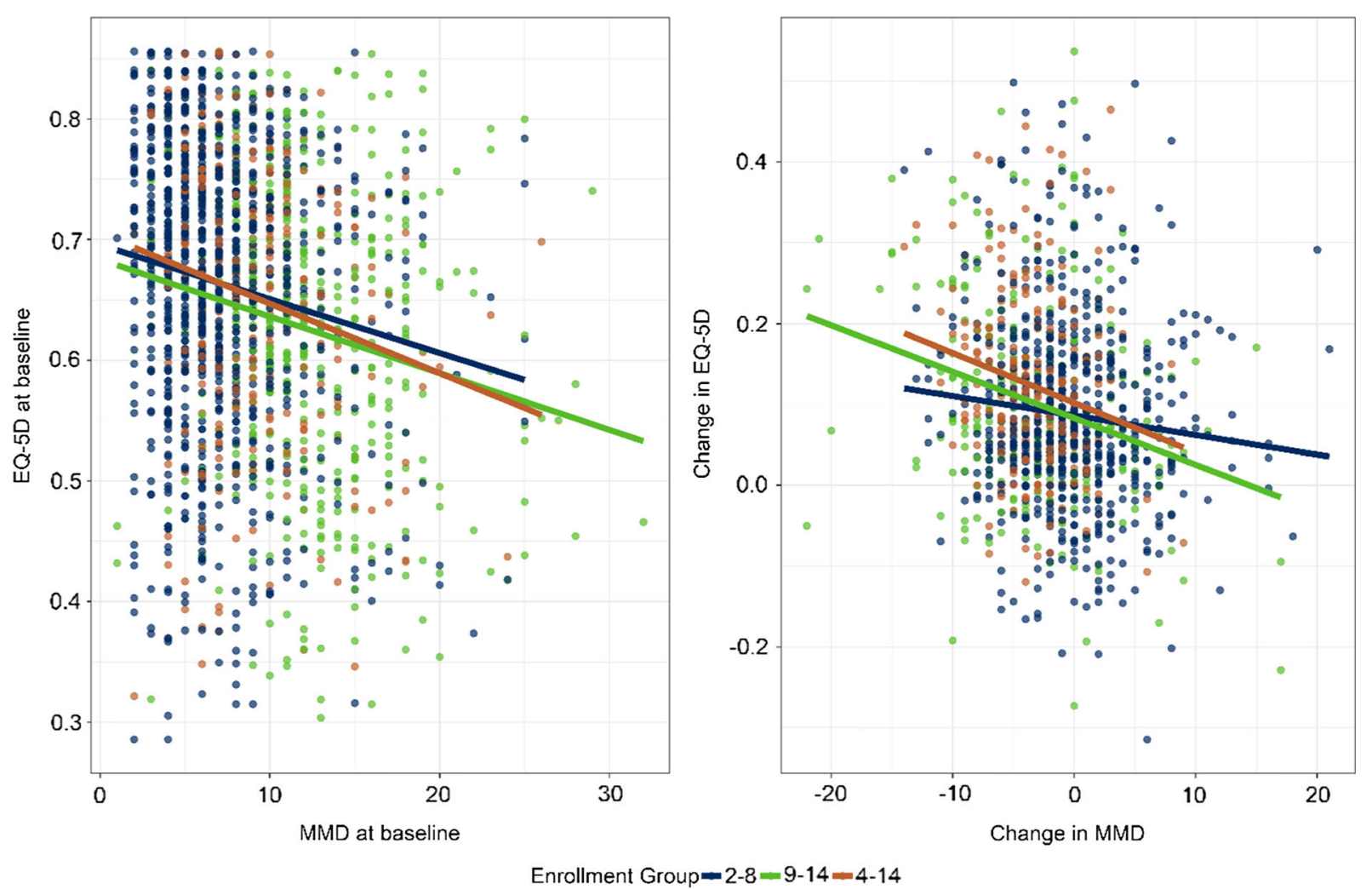

Fig. 4 Relationship between baseline absolute MMD and EQ-5D, and changes from baseline ( 52 weeks for the PRN groups and 12 weeks for the QOD + PRN group). From left to right baseline MMD versus baseline EQ-5D-3L,

analysis of erenumab for the treatment of episodic and chronic migraine, and trial-based utility values ranged from 0.773 for $4 \mathrm{MMD}$ to 0.608 for $14 \mathrm{MMD}$ on treatment with erenumab/onabotulinumtoxinA, compared to 0.759 for $4 \mathrm{MMD}$ to 0.590 for $14 \mathrm{MMD}$ while offtreatment [28]. When comparing the difference in baseline to end-of-study MMD frequency in the present analysis, and corresponding mapped EQ-5D-3L change scores, and comparing them to the difference by MMD in the erenumab/onabotulinumtoxinA analysis, the differences are more pronounced for rimegepant than for erenumab. For example, in the 4-14 QOD + PRN group, the observed change in MMD for rimegepant was approximately 9 at baseline, reduced to approximately 5.5 at endof-study (Table 2), which would be associated with a predicted utility difference of approximately +0.06 in the Lipton et al. analysis for baseline MMD versus CFB EQ-5D-3L, and CFB MMD versus CFB EQ-5D-3L. CFB change from baseline; MMD monthly migraine days

erenumab/onabotulinumtoxinA [28], while the observed difference for rimegepant was +0.12 , suggesting a stronger relationship between MMD reduction and utility improvement.

Several other studies have reported EQ-5D-3L utility scores for patients with migraine, both on days when they have migraine attacks, as well as on days when patients were migrainefree $[6,11]$. As can be expected, when pain severity decreased, utility scores increased. Reported EQ-5D-3L utility scores for when patients were migraine-free were high in these studies, ranging from 0.87 in a study from the UK [6] to 0.96 in a study from the US [11]. Possible explanations for these differences are the differences in procedures and the timing of when questionnaires are completed, because patient-reported HRQoL is influenced, among other factors, by elapsed time since the last migraine attack [14]. In the study from the UK, 
HRQoL while migraine-free was assessed directly with EQ-5D-3L within a week after a migraine attack [6], while, in the current study, it was assessed with MSQv2 at 12 weeks or longer intervals, and then mapped to EQ-5D-3L. In several recent health technology assessments for migraine prevention therapies evaluated by the National Institute of Health and Care Excellence (NICE), the mapping of MSQv2 to EQ-5D utilities was deemed more appropriate than using EQ-5D data directly, given the design of the MSQv2 instrument to be particularly relevant to migraine patients [29-31]. It may further be beneficial to capture HRQoL data more frequently to better assess change over time in future studies.

Limitations of the current analysis should be acknowledged. The mapping algorithm that was used in this study to estimate EQ-5D-3L utility values from MSQv2 has a tendency to overestimate utility values for patients with migraine who have greater disease severity [14]. The algorithm used a UK valuation set; however, Study 201 was conducted in the US, which may affect the validity of our findings. Additionally, the design of the study used as a data source for algorithm development did not allow for HRQoL assessments over time, and external validation of the algorithm using an independent data source were not conducted [14]. In the current analysis, rimegepant MSQv2 scores were based on a comparison of PRN versus QOD + PRN dosing regimens, but a placebo arm was not included, as the primary purpose of the study was long-term safety monitoring; thus, a controlled analysis of rimegepant outcomes was not feasible. However, long-term use of rimegepant QOD is currently being assessed in a phase III trial as a preventive treatment for migraine (NCT03732638), and future work will assess clinical and HRQoL benefits of rimegepant relative to placebo. In the initial 12-week results, the MSQv2 RF improved by 18.0 points (95\%CI: 15.5-20.6) in the rimegepant arm compared to 14.6 points (12.1-17.1) in the placebo arm [16]. The hierarchical nature of the analysis precluded statistical testing for this secondary outcome; however, this observed improvement is similar in magnitude to what was seen in the QOD + PRN group of Study 201 at 12 weeks [ 18.98 point increase from baseline (SD: 20.17)] [16].

\section{CONCLUSIONS}

Rimegepant $75 \mathrm{mg}$, which has been shown to be associated with reduced MMD in addition to acute treatment effects, is associated with improvement in MSQv2 domains over time, leading to estimated improvement in EQ-5D-3L utilities. While this improvement was observed in all patient-groups, it was most pronounced in those with higher MMD and those taking rimegepant on a fixed QOD plus as-needed dosing schedule.

\section{ACKNOWLEDGEMENTS}

Funding. Biohaven Pharmaceuticals funded the current analysis, BHV3000-201, and the journal's Rapid Service and Open Access fees.

Authorship. All named authors meet the International Committee of Medical Journal Editors (ICMJE) criteria for authorship for this article, take responsibility for the integrity of the work as a whole, and have given their approval for this version to be published.

Authors' Contributions. Karissa Johnston, Gilbert L'Italien, Evan Popoff, Linda Harris, and Richard B. Lipton contributed significantly to the conception and design of the work, the analysis, interpretation, and drafting of the most recently submitted version of the manuscript. Lauren Powell, Robert Croop, Alexandra Thiry, and Vladimir Coric contributed significantly to the conception of the work, the interpretation, and drafting of the most recently submitted version.

Prior Presentation. Data from this paper have been previously presented as conference posters at the following congresses: American Headache Society 2020 Virtual Annual Scientific Meeting (62nd) on June 4-7th, 2020 (abstract published in: Headache 2020;60:S1, p76), 
Academy of Managed Care Pharmacy 2020 Nexus Meeting on October 20th 2020 Virtual (abstract published in: JMCP 2020;26:10-a, pS93), and the American Academy of Neurology 2021 Virtual Annual Meeting on April 17th23rd 2021 (abstract published in: Neurology 2021; 96:15 Supplement).

Disclosures. Karissa Johnston, Evan Popoff, and Lauren Powell are employees of Broadstreet HEOR, which received funds from Biohaven for this work. Gilbert L'Italien, Robert Croop, Alexandra Thiry, and Vladimir Coric are employed by and own stock/stock options in Biohaven Pharmaceuticals. Richard B. Lipton receives research support from the NIH. He also receives support from the Migraine Research Foundation and the National Headache Foundation. He serves on the editorial board of Neurology, as a senior advisor to Headache, and as an associate editor of Cephalalgia. He has reviewed for the NIA and NINDS; holds stock options in Biohaven Holdings; and serves as consultant, advisory board member, or has received honoraria from: American Academy of Neurology, Allergan, American Headache Society, Amgen, Avanir, Biohaven, Biovision, Boston Scientific, Dr. Reddy's (Promius), Electrocore, Eli Lilly, eNeura Therapeutics, Equinox, GlaxoSmithKline, Lundbeck (Alder), Merck, Pernix, Pfizer, Supernus, Teva, Trigemina, Vector, Vedanta. He receives royalties from Oxford University Press (Wolff's Headache and Other Head Pain, 7th Edition [2001] and 8th Edition [2007]), Wiley, and Informa.

\section{Compliance with Ethics Guideli-} nes. BHV3000-201 was conducted in accordance with Good Clinical Practice, as defined by the International Conference on Harmonisation and in accordance with the ethical principles underlying European Union Directive 2001/20/EC, and the US Code of Federal Regulations, Title 21, Part 50.The protocol was approved by centralized and local Institutional Review Boards, and participants provided written informed consent before they were screened for the study. Investigators ensured that subjects, or, in those situations where consent could not be given by subjects, their legally acceptable representatives were clearly and fully informed about the purpose, potential risks, and other critical issues regarding clinical studies in which they volunteered to participate.

Data Availability. Data utilized in this study are patient-level data collected prospectively in the context of a long-term safety study. Under the ethics agreement and patient-level privacy considerations, data cannot be made directly available, but the authors can provide further details upon reasonable request.

Open Access. This article is licensed under a Creative Commons Attribution-NonCommercial 4.0 International License, which permits any non-commercial use, sharing, adaptation, distribution and reproduction in any medium or format, as long as you give appropriate credit to the original author(s) and the source, provide a link to the Creative Commons licence, and indicate if changes were made. The images or other third party material in this article are included in the article's Creative Commons licence, unless indicated otherwise in a credit line to the material. If material is not included in the article's Creative Commons licence and your intended use is not permitted by statutory regulation or exceeds the permitted use, you will need to obtain permission directly from the copyright holder. To view a copy of this licence, visit http://creativecommons.org/licenses/by$\mathrm{nc} / 4.0 /$.

\section{REFERENCES}

1. American Headache Society. AHS consensus statement: the american headache society position statement on integrating new migraine treatments into clinical practice. Headache. 2019;59:1-18.

2. Headache Classification Committee of the International Headache Society (IHS). The International Classification of Headache Disorders, 3rd edition. Cephalalgia. 2018;38(1):1-211.

3. Mayans L, Walling A. Acute Migraine headache: treatment strategies. Am Fam Physician. 2018;97(4):243-51. 
4. Doane M, Gupta S, Fang J, et al. The humanistic and economic burden of migraine in Europe: a crosssectional survey in five countries. Neurol Ther. 2020;2020:1-15.

5. Doane M, Gupta S, Vo P, et al. Associations between headache-free days and patient-reported outcomes among migraine patients: a cross-sectional analysis of survey data in Europe. Pain Ther. 2019;8(2): 203-16.

6. Stafford M, Hareendran A, Ng-Mak D, Insinga R, Xu $R$, Stull D. EQ-5D ${ }^{\mathrm{TM}}$-derived utility values for different levels of migraine severity from a UK sample of migraineurs. Health Qual Life Outcomes. 2012;10:65.

7. Blumenfeld A, Varon S, Wilcox T, et al. Disability, HRQOL and resource use among chronic and episodic migraineurs: results from the International Burden of Migraine Study (IBMS). Cephalalgia. 2011;31(3):301-15.

8. Ford JH, Jackson J, Milligan G, Cotton S, Ahl J, Aurora SK. A real-world analysis of migraine: a cross-sectional study of disease burden and treatment patterns. Headache. 2017;57(10):1532-44.

9. Buse DC, Rupnow MF, Lipton RB. Assessing and managing all aspects of migraine: migraine attacks, migraine-related functional impairment, common comorbidities, and quality of life. Mayo Clin Proc. 2009;84(5):422-35.

10. Lampl C, Thomas H, Stovner LJ, Tassorelli C, Katsarava Z, Laínez JM, et al. Interictal burden attributable to episodic headache: findings from the Eurolight project. J Headache Pain. 2016;17:9.

11. $\mathrm{Xu} \mathrm{R}$, Insinga R, Golden $\mathrm{W}, \mathrm{Hu} X$. EuroQol (EQ-5D) health utility scores for patients with migraine. Qual Life Res. 2011;20:601-8.

12. Diener H-C, Tassorelli C, Dodick DW, Silberstein SD, Lipton RB, Ashina M, et al. Guidelines of the International Headache Society for controlled trials of preventive treatment of migraine attacks in episodic migraine in adults. Cephalalgia. 2020;40(10): 1026-44.

13. Tassorelli C, Diener HC, Dodick DW, Silberstein SD, Lipton RB, Ashina $M$, et al. Guidelines of the International Headache Society for controlled trials of preventive treatment of chronic migraine in adults. Cephalalgia. 2018;38(5):815-32.

14. Gillard P, Devine B, Varon S, Liu L, Sullivan S. Mapping from disease-specific measures to healthstate utility values in individuals with migraine. Value in Health. 2012;15(3):485-95.
15. Croop R, Goadsby P, Stock D, et al. Efficacy, safety, and tolerability of rimegepant orally disintegrating tablet for the acute treatment of migraine: a randomised, phase 3, double-blind, placebo-controlled trial. The Lancet. 2019;394(10200):737-45.

16. Croop R, Lipton RB, Kudrow D, Stock DA, Kamen L, Conway $\mathrm{CM}$, et al. Oral rimegepant for preventive treatment of migraine: a phase $2 / 3$, randomised, double-blind, placebo-controlled trial. The Lancet. 2020;397(10268):51-60.

17. Harris L, L'Italien G, Croop R, Stock E, Thiry A, Cowrie $\mathrm{K}$, et al. Acute treatment of migraine with oral rimegepant $75 \mathrm{mg}$ improves health related quality of life: results from a long-term, open-label safety study (BHV3000-201)(1943). Neurology. 2020;94:1943.

18. Lipton R, Berman G, Kudrow D, Mullin K, Thiry A, Lovegren $\mathrm{M}$, et al. Long-Term, Open-Label Safety Study of Rimegepant $75 \mathrm{mg}$ for the Treatment of Migraine (Study 201): Interim Analysis of Safety and Exploratory Efficacy. In: American Headache Society 61st Annual Scientific Meeting; July 11-14; Philadelphia, PA: AHS; 2019.

19. McGinley J, L'Italien G, Thiry A, Croop R, Coric V, Lipton R. Rimegepant $75 \mathrm{mg}$ results in reductions in monthly migraine days: secondary analysis of a multicenter, open label long-term safety study of rimegepant for the acute treatment of migraine. Neurology. 2020;94:1793.

20. Headache Classification Committee of the International Headache Society (IHS). The International Classification of Headache Disorders, 3rd edition (beta version). Cephalalgia Int $\mathrm{J}$ Headache. 2013;33(9):629-808.

21. Bagley C, Rendas-Baum R, Maglinte G, Yang M, Varon S, Lee J, et al. Validating Migraine-Specific Quality of Life Questionnaire v2.1 in episodic and chronic migraine. Headache. 2012;52(3):409-12.

22. Jhingran P, Osterhaus J, Miller D, Lee J, Kirchdoerfer L. Development and validation of the migrainespecific quality of life questionnaire. Headache. 1998;38(4):295-302.

23. Speck R, Shalhoub H, Ayer D, Ford J, Wyrwich K, Bush E. Content validity of the Migraine-Specific Quality of Life Questionnaire version 2.1 electronic patient-reported outcome. J Patient-Report Outcomes. 2019;3:39.

24. Glaxo Welcome Inc. Migraine specific quality of life questionnaire (version 2.1). ePROVIDE; 1998.

25. Cole JC, Lin P, Rupnow MF. Minimal important differences in the Migraine-Specific Quality of Life 
Questionnaire (MSQ) version. Cephalalgia. 2009;29(11):1180-7.

26. EuroQoL Research Foundation. EQ-5D-3L User Guide 2018. 2018. https://euroqol.org/ publications/user-guides.

27. Di Tana G, Porter J, Lipton R, Hatswell A, Sapra S, Villa G. Longitudinal assessment of utilities in patients with migraine: an analysis of erenumab randomized controlled trials. Health Qual Life Outcomes. 2019;17:171.

28. Lipton R, Brennan A, Palmer S, Hatswell A, Porter J, Sapra $S$, et al. Estimating the clinical effectiveness and value-based price range of erenumab for the prevention of migraine in patients with prior treatment failures: a US societal perspective. J Med Econ. 2018;2018:1-10.

29. NICE. Fremanezumab for preventing migraine: technology appraisal guidance [TA631]. 2020. https://www.nice.org.uk/guidance/ta631/evidence. https://www.nice.org.uk/guidance/ta631. Accessed 3 Jun 2020.

30. NICE. Galcanezumab for preventing migraine: Technology appraisal guidance [TA659]. 2020. https://www.nice.org.uk/guidance/ta659. https:// www.nice.org.uk/guidance/ta659. Accessed 18 Nov 2020 .

31. NICE. Erenumab for preventing migraine: Technology appraisal guidance [TA682]. 2021. https:// www.nice.org.uk/guidance/ta682/evidence. 2021. 